\title{
EL EMPRENDIMIENTO POR NECESIDAD UN FACTOR QUE INCIDE EN EL DESEMPLEO JUVENIL EN ESTUDIANTES DE LA FACULTAD DE CIENCIAS EMPRESARIALES, 2015
}

\section{Entrepreneurship in need a factor affecting youth unemployment in students of the Faculty of Business, 2015}

\section{Luis Catacora Lira ${ }^{6}$}

\section{RESUMEN}

Objetivo: El trabajo de investigación tuvo como objetivo principal el determinar la incidencia del desempleo por necesidad en el inicio y desarrollo de los emprendimientos en los estudiantes de la Facultad de Ciencias Empresariales en el año 2015.

Método: Para el desarrollo del estudio, se vio por conveniente aplicar el diseño de investigación descriptiva explicativa, no experimental. La muestra de estudio estuvo compuesta por los estudiantes del V y VII ciclo de la escuela Profesional de Ingeniería Comercial de la Facultad de Ciencias Empresariales. Se preparó dos cuestionarios orientados a recoger información de cada una de las variables, respectivamente validado. Los datos fueron procesados con el programa estadístico SPSS, y los análisis estadísticos son descriptivos e inferenciales.

Resultado: El problema del desempleo es una preocupación que todos los gobiernos buscan resolver. El desempleo juvenil es actualmente uno de los temas que requiere tratamiento por encontrarse en aumento, y afecta a un significativo

${ }^{6}$ Magister en Docencia Universitaria, candidato a Doctor en Ciencias de la Educación. número de jóvenes. En ese sentido el presente artículo de investigación brinda una explicación sobre una de las causas del surgimiento y crecimiento del desempleo juvenil. El tema trasciende el pensamiento clásico que sostiene que el desempleo juvenil es producto de la escasa inversión que realizan las empresas en la economía. Porque la investigación ha permitido modificar esa tesis, y ahora sostener que el desempleo juvenil es producto en gran medida de la improvisación empresarial y desconocimiento de teorías económicas básicas por parte de los jóvenes que se lanzan por primera vez al mercado con un emprendimiento.

Conclusiones: En ese contexto el presente estudio logró determinar que el emprendimiento por necesidad, tiene incidencia en el surgimiento y crecimiento del desempleo juvenil en la región de Tacna. Iniciar emprendimientos en economías competitivas y mercados impredecibles, requiere de preparación empresarial, por lo menos en lo fundamental; no es viable ni recomendable pretender iniciar un negocio con el sólo propósito de probar suerte. Se requiere actualmente de mucho conocimiento y entrenamiento económico, financiero y comercial.

\section{PALABRAS CLAVES}

Empleo informal, Emprender, Negocio. 


\section{ABSTRAC}

Objective: The research's main objective was to determine the incidence of unemployment by necessity in the initiation and development of entrepreneurship in students from the School of Business Administration in 2015.

Method: For the development of the study, was appropriate to apply for the design of explanatory descriptive, not experimental. The study sample consisted of students of the V and VII cycle of the Professional School of Business Administration School of Business. Two questionnaires designed to collect information from each of the variables respectively prepared validated. The data were processed using the SPSS statistical program, and statistical analysis are descriptive and inferential.

Result: The problem of unemployment is a concern for all governments seeks to solve. Youth unemployment is currently one of the issues that require treatment because they were on the rise and affects a significant number of young people. In that sense this research paper provides an explanation of the causes of the emergence and growth of youth unemployment. The issue transcends the classical thought that argues that youth unemployment is a result of the low investment made by companies in the economy. Because research has allowed to modify the thesis, and now argue that youth unemployment is the result largely of corporate improvisation and basic ignorance of economic theory by young people who rush first to market with a venture.

Conclusions: In this context the present study was determined that entrepreneurship by necessity, has an impact on the emergence and growth of youth unemployment in the region of Tacna. Log enterprises in competitive economies and unpredictable markets, business require preparation, at least in substance; It is neither feasible nor advisable intending to start a business with the sole purpose to try their luck. It currently requires a lot of knowledge and economic, financial and commercial training.

\section{KEYWORDS}

Informal Entrepreneurship, Business

\section{INTRODUCCIÓN}

Actualmente las Universidades vienen formando profesionales para que resuelvan los principales problemas que tiene la sociedad peruana. Pero también existen una fuerte cantidad de jóvenes que se encuentran excluidos del sistema universitario. Es decir, se encuentran en algún lugar buscando la manera de emprender un negocio. Se conoce que el empleo depende de la inversión. Pero no cualquier inversión es garantía de empleo. Muchas veces se piensa que al iniciar un negocio ya se acabó el desempleo. La realidad nos indica que existe una elevada tasa de mortalidad de pequeños negocios. En ese sentido iniciar un emprendimiento no siempre es sinónimo de empleo.

La economía peruana requiere de jóvenes emprendedores que impulsen el crecimiento económico. Existen generaciones de jóvenes que tienen excelentes ideas de negocio, que son potenciales inversores que de su adecuada y 
pertinente participación en el mercado dependerá el progreso y mejoramiento de la competitividad del país. Pero también existen jóvenes que desligados de una formación empresarial se inician en un emprendimiento pero por necesidad, que los induce a correr un alto riesgo. Se considera dentro de la teoría económica que para asegurar el éxito de un emprendimiento se tiene que realizar un conjunto de estudios, que va desde un estudio de mercado hasta una evaluación económica financiera.

La presente investigación permitió conocer que el emprendimiento por necesidad, no siempre es garantía de éxito, que es indispensable que los jóvenes emprendedores reciban capacitaciones en el campo empresarial que les permita reducir el riesgo y fortalecer su confianza los resultados de la inversión.

En el campo empresarial es fundamental que los estudiantes que se formen para ser emprendedores, se preparen para contrarrestar el crecimiento de la tendencia del desempleo juvenil. El emprendimiento debe ser una cualidad de todo estudiante con formación empresarial. La iniciativa, la creatividad y la innovación en el campo empresarial, es fundamental para el crecimiento económico.

\section{1.- EMPRENDIMIENTO POR NECESIDAD}

Emprender por necesidad es poner en marcha una idea de negocio de forma apresurada sin conocer si tiene o no el potencial de mercado para generar ingresos. Quienes emprenden bajo esta modalidad se lanzan a la aventura empresarial por solucionar situaciones financieras personales, porque han perdido su trabajo 0 se encuentran frustrados en su ámbito laboral. Generalmente, este tipo de emprendimientos depende solo del emprendedor, el cual se convierte en un empleado de tiempo completo.

El emprendimiento por necesidad surge desde el momento en que la persona se siente incapaz de integrarse a cualquier organización establecida. Bien sea porque no encuentra una oportunidad laboral, o porque no logra conciliar vida personal con la vocación o porque se queda fuera del mercado laboral competitivo. Al respeto Jurgen Weller (2006) sostiene: Las mujeres jóvenes están desarrollando en forma cada vez más marcada un desarrollo personal autónomo, y para independizarse de los roles tradicionales estrechamente vinculados con el hogar el empleo juega un papel clave. Sin embargo, se enfrentan a problemas especiales de inserción laboral siendo este problema especialmente grave para mujeres con educación formal baja, para las cuales hay muy pocas oportunidades de empleo productivo.

Las características comunes de los negocios surgidos de la necesidad son:

a) Cuentan con ingresos más bajos que los que emprenden por oportunidad.

b) El fundador suele tener más edad que en los casos del emprendedor vocacional, de media, 1,5 años mayor.

c) Tienen estudios medios y superiores, aunque en este concepto están por debajo de los de oportunidad.

d) Suelen poseer más experiencia profesional en el sector y empiezan con un capital semilla que ronda los 500 soles. 


\section{2.- $\quad$ EL DESEMPLEO JUVENIL}

El acceso al empleo constituye para la mayor parte de los jóvenes un símbolo de mayoría de edad, puesto que el ingreso al mundo del trabajo representa, ante todo, la transición a la vida adulta. Sin embargo, la falta de empleo es un problema que afecta especialmente a los jóvenes, que se ven expuestos, en comparación con los adultos, a mayores niveles de incertidumbre económica y social.

Actualmente hay en el mundo unos 1.200 millones de jóvenes, de edades comprendidas entre los 15 y los 24 años y que constituyen el $17 \%$ de la población mundial. Como ha indicado la Organización Internacional del Trabajo "están llamados a prestar una contribución importante como trabajadores productivos, empresarios, consumidores, ciudadanos, miembros de la sociedad civil y agentes del cambio. Su energía y capacidad de innovación son recursos inestimables que ningún país puede permitirse desperdiciar".

Sin embargo, necesitan un apoyo social decidido para conseguirlo, porque "son también extremadamente vulnerables. Afrontan un alto grado de incertidumbre económica y social. Con demasiada frecuencia, no se aprovechan todas sus potencialidades porque no tienen acceso a empleos productivos y decentes".

La crisis económica ha llevado a que esa protección haya disminuido dramáticamente en muchos lugares del mundo, con consecuencias negativas para el conjunto de la sociedad.

El desempleo de los jóvenes presentan las siguientes características:

a) Los jóvenes conforman un grupo poblacional particularmente afectado por el problema del desempleo.

b) Los jóvenes sufren una mayor incertidumbre económica y social, que tiende a expresarse en una mayor vulnerabilidad al desempleo. La probabilidad de los jóvenes de estar desempleados es 3 veces mayor que la de los adultos. Son los más jóvenes entre los jóvenes los más vulnerables al desempleo: la probabilidad de los adolescentes de estar desempleados es 4 veces mayor que la de los adultos.

c) El desempleo juvenil se concentra en los grupos sociales más vulnerables: entre los jóvenes son las mujeres y los menos educados los más afectados por el desempleo. A su vez, la mayor parte de los jóvenes desempleados provienen de hogares de escasos recursos, muchos de los cuales se encuentran en situación de pobreza. Se genera con ello un círculo vicioso de transmisión intergeneracional de la pobreza.

d) Los jóvenes no enfrentan mayores dificultades de acceso al empleo que las halladas por los adultos. La permanencia de los jóvenes en situaciones de desempleo es similar o menor que la observada en los adultos. Por consiguiente, el elevado desempleo juvenil no se explica por la presencia de barreras generalizadas a la "entrada al empleo".

e) La temprana e inestable inserción en el mundo del trabajo constituye el principal determinante del desempleo juvenil. Las trayectorias laborales hacia el desempleo son comparativamente más frecuentes entre los jóvenes, ya sea por la pérdida del empleo o por la incorporación al mercado de trabajo desde situaciones de inactividad. 
f) Se identifican tres grupos de jóvenes sin empleo cuya situación sociolaboral requiere atención prioritaria en materia de política social y de empleo. Ellos son: a) los adolescentes desempleados que no estudian, b) los jóvenes desempleados con responsabilidades familiares y los adolescentes que no trabajan, no buscan trabajo ni estudian.

El desempleo juvenil y las situaciones que llevan a los jóvenes a renunciar a la búsqueda de empleo o a trabajar en condiciones inadecuadas suponen un coste para la economía, la sociedad, el individuo y su familia. La falta de trabajo decente, si se experimenta a temprana edad, puede representar una amenaza para las perspectivas laborales futuras de una persona y suele suponer patrones de comportamiento laboral inapropiados que perduran toda la vida. Las sociedades pierden lo que han invertido en la educación y los gobiernos reducen sus ingresos al sistema de seguridad social y se ven obligados a gastar más en políticas sociales, como los programas de prevención del uso de drogas y el crimen. Todo ello amenaza el potencial de desarrollo de las economías. Por lo tanto, tiene sentido para un país centrarse en la juventud desde un punto de vista del análisis coste-beneficio. Puede que los jóvenes no tengan experiencia, pero tienden a estar muy motivados y son capaces de ofrecer nuevas ideas o planteamientos. Son los impulsores del desarrollo económico de un país, por lo que privarse de ese potencial es una pérdida económica.

Los informes de la OIT también han revelado que existe un vínculo comprobado entre el desempleo juvenil y la exclusión social. "La incapacidad de encontrar empleo genera una sensación de inutilidad y ociosidad entre los jóvenes, y puede elevar los índices de criminalidad, problemas de salud mental, violencia, conflictos y consumo de drogas.

En ese sentido, la ausencia de responsabilidades profesionales y familiares, la falta de motivación para comportarse correctamente y el exceso de tiempo libre se convierten en un cóctel explosivo que facilita un comportamiento irregular, por lo que algunos estudios relacionan el aumento del desempleo con el de los delitos, especialmente los que atentan a la propiedad y los relacionados con el tráfico de drogas, con la consiguiente pérdida de futuro para los que los cometen.

\section{METODOLOGÍA}

La presente investigación es descriptiva explicativa, con un diseño no experimental, orientado a determinar la incidencia del emprendimiento por necesidad en el desempleo juvenil en los estudiantes del V y VII ciclo de la Escuela Profesional de Ingeniería Comercial de la Facultad de Ciencias Empresariales. Para el análisis estadístico las variables fueron operacionalizadas considerando su naturaleza cualitativa. Fueron categorizadas y medidas a través de escalas de valoración. El proceso de planificación y diseño de los instrumentos fueron desarrollados en base a las dimensiones e indicadores que fundamenta las bases teóricas de cada variable. Las variables el emprendimiento por necesidad y desempleo juvenil, fueron analizadas en forma parcial para luego realizar una evaluación total, para determinar los niveles que busca los objetivos de la investigación. En este proceso de investigación se utilizó la técnica de la encuesta, para lo cual se elaboraron dos cuestionarios con enunciados orientados a obtener los datos exigidos por el estudio, con 
respuestas que estaban categorizadas con la escala de Lickert, con posesiones que van de totalmente cierto al no es cierto. Los puntajes fluctuaban entre (1) y (4). Los indicadores de cada una de las variables permitieron hacer viable la elección de los modelos estadísticos adecuados a la naturaleza de las variables. El cuestionario se administró a los estudiantes del V y VII ciclo de estudios. EI nivel de fiabilidad según el coeficiente de Alpha de Cronbach para la variable emprendimiento por necesidad fue de (0.785) y para la variable desempleo juvenil fue de $(0.768)$ valores que superan el valor $(0.70)$ por la cual son válidos para la investigación.

Para el estudio se eligió una muestra representativa de 60 estudiantes, todos ellos matriculados en la carrera profesional con asistencia regular y promedio académico satisfactorio. Los criterios de selección fueron de conveniencia y de expertos, considerando la naturaleza heterogeneidad de la población.

Los datos obtenidos de la aplicación de los cuestionarios, fueron organizados, clasificados y procesados en tablas y gráficos estadísticos, considerando la naturaleza de cada variable. En este caso se trata de variables cualitativas nominales que fueron evaluadas con escalas de valoración. Para efectos del análisis de los datos se vio por conveniente combinar los métodos de análisis cualitativos y cuantitativos, combinando el análisis estadístico descriptivo e inferencial, sin dejar de realizar el análisis estadístico por cada variable, es decir, por cada una de las categorías que se formaron por la relación entre las variables.

Para la comprobación de hipótesis, implicó la estimación de los coeficientes de correlación V de Cramer y el coeficiente PHI. Asimismo se vio por conveniente aplicar las pruebas del $\mathrm{CHI}$ cuadrado y la prueba "t" de student, para brindar el nivel de confianza del $95 \%$ a los resultados de la investigación. Las pruebas de análisis correlacional y de prueba de hipótesis se realizó con cada de las variables, de tal manera que el estudio refleje en primer lugar los resultados parciales y en segundo lugar el resultado final de la investigación.

Se administró el Programa SPSS versión 15 para el análisis de variables cualitativas convertidas a la escala numérica.

\section{RESULTADOS}

\section{1.- ANÁLISIS ESTADÍSTICO DEL EMPRENDIMIENTO POR NECESIDAD}

\section{1.- ANÁLISIS DE CONFIABILIDAD DEL INSTRUMENTO}

\begin{tabular}{|c|c|}
\hline $\begin{array}{c}\text { Alfa de } \\
\text { Cronbach }\end{array}$ & $\begin{array}{c}\mathrm{N} \text { de } \\
\text { elementos }\end{array}$ \\
\hline 0.785 & 30 \\
\hline
\end{tabular}

El coeficiente de Alpha de Cronbach de 0.785 , significa que el instrumento sobre emprendimiento por necesidad, es fuertemente confiable. 


\section{2.- PRUEBA DE HIPÓTESIS SOBRE EMPRENDIMIENTO POR NECESIDAD}

Para establecer un nivel de confianza sobre los resultados del análisis estadístico, se desarrolla la siguiente prueba de hipótesis para la media de las respuestas, considerando la escala de valoración y las siguientes hipótesis estadísticas:

\section{Escala de valoración}

\begin{tabular}{|l|c|}
\hline Niveles de aceptación & Puntajes \\
\hline Alto nivel de aceptación & $59-80$ \\
\hline Moderado nivel de aceptación & $38-58$ \\
\hline Bajo nivel de aceptación & $16-37$ \\
\hline
\end{tabular}

\section{HIPÓTESIS ESTADÍSTICAS}

$$
\begin{aligned}
& \mathrm{H}_{0}: \quad \mu<58 \quad \text { Bajo nivel de aceptación del } \\
& \mathrm{H}_{1}: \quad \mu \geq 58 \begin{array}{c}
\text { Alto nivel de aceptación del } \\
\text { Alsprentimiento por necesidad }
\end{array} \\
& \alpha=5 \% \quad \text { Nivel de significación } \\
& g l=n-1=30-1=29
\end{aligned}
$$

Se reemplaza en la siguiente fórmula de la distribución "t" los datos obtenidos del análisis estadístico, y se procede a ejecutar la prueba de hipótesis para la media de la siguiente forma:

\begin{tabular}{|l|c|}
\hline Estadísticos & Valores \\
\hline $\begin{array}{l}\text { Sumatoria de las medias del total de los } \\
\text { ítems }\end{array}$ & 53.78 \\
\hline $\begin{array}{l}\text { Sumatoria de las desviaciones típicas del } \\
\text { total de ítems }\end{array}$ & 16.17 \\
\hline
\end{tabular}

Formula de la prueba " $\mathrm{t}$ ".

$$
\begin{gathered}
\mathrm{t}=\frac{\mathrm{X}-\mu}{\mathrm{S} / \sqrt{\mathrm{n}}} \\
\mathrm{t}=\frac{53.78-58}{16.17 / \sqrt{ } 30}
\end{gathered}
$$

Se tiene que el valor de $\mathrm{t}=-1.43$ 


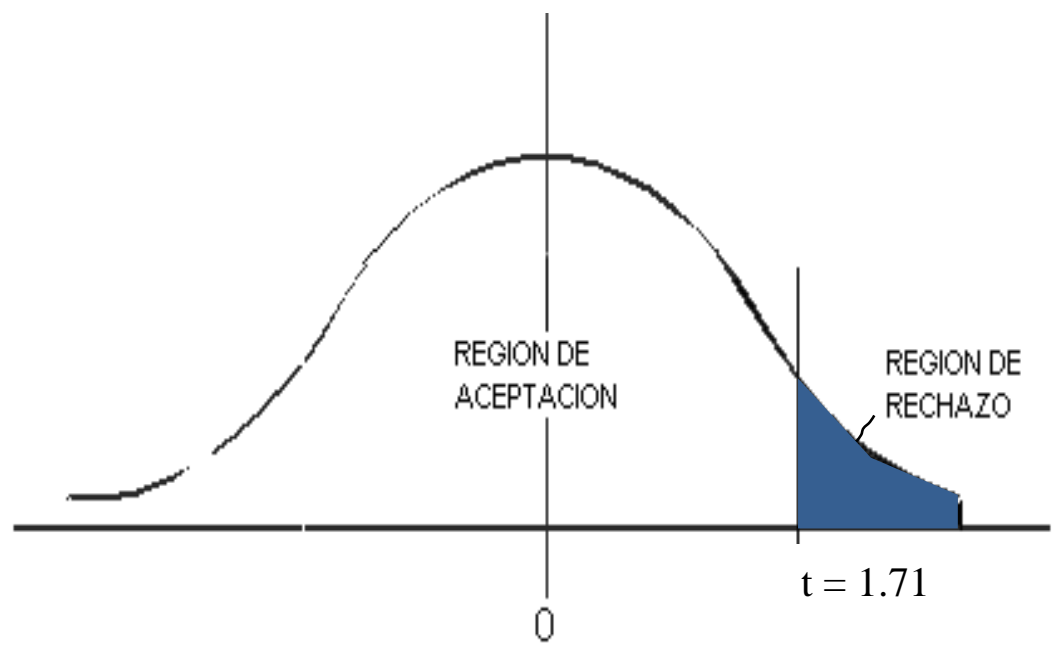

\section{Decisión}

El valor de " $t_{c}$ " calculado (- 1.43) cae en la región de aceptación, por lo tanto se acepta la hipótesis nula $\left(\mathrm{H}_{0}\right)$ y se rechaza la hipótesis alternativa.

\section{Conclusión}

Se concluye con un nivel de confianza del $95 \%$, que los estudiantes expresan un bajo nivel de aceptación de que se produzca un emprendimiento por necesidad en los estudiantes que tienen estudios empresariales.

Por lo tanto, se procede a ACEPTAR la hipótesis Nula de la investigación planteada.

\section{2.- $\quad$ ANÁLISIS ESTADÍSTICO DEL DESEMPLEO JUVENIL}

\section{1.- ANÁLISIS DE CONFIABILIDAD DEL INSTRUMENTO}

\begin{tabular}{|c|c|}
\hline $\begin{array}{c}\text { Alfa de } \\
\text { Cronbach }\end{array}$ & $\begin{array}{c}\mathrm{N} \text { de } \\
\text { elementos }\end{array}$ \\
\hline 0.768 & 30 \\
\hline
\end{tabular}

El coeficiente de Alpha de Cronbach de 0.768 , significa que el instrumento sobre emprendimiento por necesidad, es fuertemente confiable. 


\section{2.- PRUEBA DE HIPÓTESIS SOBRE DESEMPLEO JUVENIL}

Para establecer un nivel de confianza sobre los resultados del análisis estadístico, se desarrolla la siguiente prueba de hipótesis para la media de las respuestas, considerando la escala de valoración y las siguientes hipótesis estadísticas:

Escala de valoración

\begin{tabular}{|l|c|}
\hline $\begin{array}{l}\text { Niveles de aceptación del desempleo } \\
\text { juvenil }\end{array}$ & Puntajes \\
\hline Alto nivel de aceptación & $59-80$ \\
\hline Moderado nivel de aceptación & $38-58$ \\
\hline Bajo nivel de aceptación & $16-37$ \\
\hline
\end{tabular}

\section{HIPÓTESIS ESTADÍSTICAS}

$$
\begin{aligned}
& \mathrm{H}_{0}: \quad \mu<58 \text { Bajo nivel de aceptación del desempleo } \\
& \mathrm{H}_{1}: \mu \geq 58 \text { Alto nivel de aceptación del desempleo } \\
& \text { juvenil } \\
& \alpha=5 \% \quad \text { Nivel de significación } \\
& g l=n-1=30-1=29
\end{aligned}
$$

Se reemplaza en la siguiente fórmula de la distribución " $\mathrm{t}$ " los datos obtenidos del análisis estadístico, y se procede a ejecutar la prueba de hipótesis para la media de la siguiente forma:

\begin{tabular}{|l|c|}
\hline Estadísticos & Valores \\
\hline $\begin{array}{l}\text { Sumatoria de las medias del total de los } \\
\text { ítems }\end{array}$ & 56.17 \\
\hline $\begin{array}{l}\text { Sumatoria de las desviaciones típicas del } \\
\text { total de ítems }\end{array}$ & 16.16 \\
\hline
\end{tabular}

Formula de la prueba " $\mathrm{t}$ ".

$$
\begin{aligned}
& \mathrm{t}=\mathrm{X}-\mu \\
& \mathrm{S} / \sqrt{\mathrm{n}} \\
& \mathrm{t}=\frac{56.17-58}{16.16 / \sqrt{ } 30}
\end{aligned}
$$

Se tiene que el valor de $t=-0.62$ 


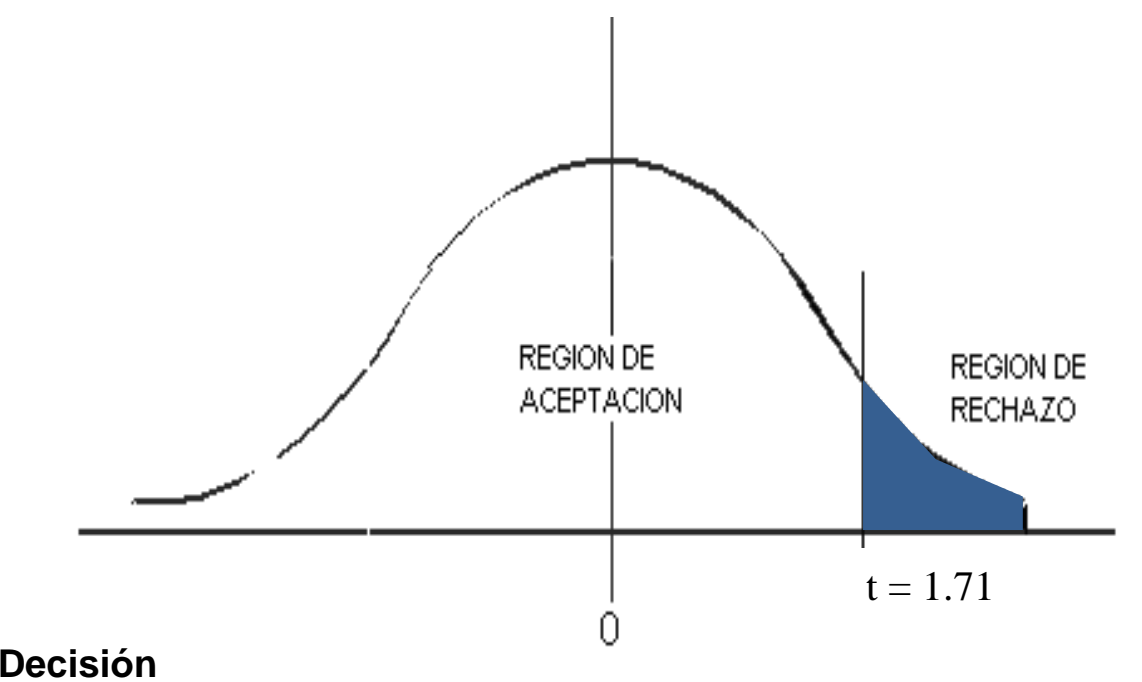

El valor de " $\mathrm{t}_{\mathrm{c}}$ " calculado ( - 0.62) cae en la región de aceptación, por lo tanto se acepta la hipótesis nula $\left(\mathrm{H}_{0}\right)$ y se rechaza la hipótesis alternativa.

\section{Conclusión}

Se concluye con un nivel de confianza del 95\%, que los estudiantes expresan un bajo nivel de aceptación de que se produzca el desempleo juvenil en personas que tienen estudios empresariales.

Por lo tanto, se procede a ACEPTAR la hipótesis Nula de la investigación planteada.

\section{3.- PRUEBA ESTADISTICA DE RELACIÓN ENTRE EMPRENDIMIENTO POR NECESIDAD Y DESEMPPLEO JUVENIL}

Tabla № 1: Relación entre emprendimiento y desempleo juvenil

\begin{tabular}{|ll|c|c|}
\hline Correlaciones & & Valor & $\begin{array}{c}\text { Sig. } \\
\text { aproximada }\end{array}$ \\
\hline Nominal por & Phi & .435 & .225 \\
nominal & V de Cramer & .307 & .225 \\
& Coeficiente de & .399 & .225 \\
N de casos válidos & & 30 & \\
\hline
\end{tabular}

a) Asumiendo la hipótesis alternativa.

b) Empleando el error típico asintótico basado en la hipótesis nula. 


\section{Decisión}

El valor del coeficiente de correlación Phi (0.435), y V de Cramer (0.307), se ubican en el nivel de correlación moderada.

\section{Conclusión}

Se concluye que el nivel de emprendimiento por necesidad y el nivel de desempleo juvenil, están moderadamente asociadas, lo que significa que los estudiantes que emprendan un negocio por necesidad sin tener conocimientos empresariales presentan un alto riesgo de fracasar y permanecer desempleado respecto de aquellos que tienen estudios empresariales.

Tabla $\mathbf{N}^{\circ}$ 2: Prueba estadística del Chi cuadrado entre emprendimiento por necesidad y desempleo juvenil.

Pruebas de chi-cuadrado

\begin{tabular}{|c|c|c|c|}
\hline & Valor & $\mathrm{gl}$ & $\begin{array}{l}\text { Sig. asintótica } \\
\text { (bilateral) }\end{array}$ \\
\hline Chi-cuadrado de Pearson & $5.667^{a}$ & 4 & .225 \\
\hline Razón de v erosimilitudes & 6.047 & 4 & .196 \\
\hline $\begin{array}{l}\text { Asociación lineal por } \\
\text { lineal }\end{array}$ & 5.293 & 1 & .021 \\
\hline N de casos válidos & 30 & & \\
\hline
\end{tabular}

a. 9 casillas $(100.0 \%)$ tienen una frecuencia esperada inferior a 5. La f recuencia mínima esperada es 2.70.

\section{Análisis de datos}

Hipótesis de investigación:

$\left(\mathrm{H}_{0}\right)$ : $\quad$ El emprendimiento por necesidad y el desempleo juvenil; son independientes.

$\left(H_{1}\right)$ : $\quad$ El emprendimiento por necesidad y el desempleo juvenil; son dependientes.

Regla de decisión

$\mathrm{Si}$ valor Sig. $>0.05$

$\mathrm{Si}$ valor Sig $<0.05$

entonces se acepta la hipótesis Nula

entonces se acepta la hipótesis alternativa $\left(\mathrm{H}_{1}\right)$

\section{Decisión}

Como el valor de sig. Asintótica es de 0.225 es mayor que el valor teórico 0.05 , entonces se acepta la hipótesis nula $\left(\mathrm{H}_{0}\right)$ y se rechaza la hipótesis alternativa.

\section{Conclusión}

Se concluye que los inversores que se inicien en determinados emprendimientos por necesidad, estan expuestos en mayor medida de perder el empleo productivo que aquellos que tienen estudios empresariales, con un nivel de confianza del $95 \%$. 


\section{CONCLUSIONES}

a) La tendencia de los jóvenes de lanzarse al mercado en un acto de emprender por necesidad, sin tener conocimientos empresariales, implica una alta probabilidad de fracasar en el corto plazo.

b) El desempleo juvenil es consecuencia de la improvisación y desconocimiento del comportamiento de las variables que inciden directamente en todo tipo de emprendimiento.

c) El nivel de competitividad y productividad de las microempresas que tienen que desarrollar para permanecer en el mercado, establece condiciones de empleo calificado que induce al desempleo de los jóvenes sin preparación empresarial.

d) El emprendimiento por necesidad, que surge de la necesidad de trabajar para mantener una familiar, es un problema social en la que están involucrados los jóvenes de 18 a los 24 años de edad.

e) La inmadurez de muchos jóvenes que tempranamente forman familias por muchas razones, se ven en la obligación de arriesgar todo lo que tienen, sin prever de los conocimientos empresariales que deben tener para reducir su riesgo económico financiero.

f) El emprendimiento por necesidad y el desempleo juvenil son dos factores críticos que afectan el crecimiento económico del país.

g) En la medida en que los jóvenes sin estudios empresariales inicien emprendimientos por necesidad económica, incrementará la tendencia del desempleo juvenil.

h) Con un nivel de confianza del $95 \%$ se confirma que el emprendimiento por necesidad de los jóvenes tiene incidencia en el incremento del desempleo juvenil.

\section{REFERENCIAS BIBLIOGRÁFICAS}

- ALLES, Martha Alicia (2000) Dirección Estratégica de Recursos Humanos: Gestión por Competencias.

- BENAVIDES ESPÍNDOLA Olga (2002) Competencias y competitividad: diseño para organizaciones latinoamericanas. Colombia Mc. Graw Hill $p$ 145.

- CATACORA LIRA, Luis. (2012). Fundamentos para investigar y presentar una tesis. Tacna. UPT. Fondo Editorial.

- CAMPOS RÍOS, Guillermo. (2008). La economía informal y sus posibilidades de desarrollo. Disponible: http://www.ucm.es/info/ec/ecocri/eus/campos_rios.pdf; Consultado: 161011.

- DÍAZ, C. M. \& Celis, J. E. (2011). La formación para el trabajo en la educación media en Colombia. Revista Latinoamericana de Ciencias Sociales, Niñez y Juventud, 1 (9), pp. 371 - 380.

- MANUAL DE CONSTITUCION DE MICROEMPRESAS. Disponible. http://www.microjustice4all.org/web/pdf/AnexosP/6_8.pdf. Consultado: 14 de octubre 2011. 
- MÜNICH, L. (1996). Más allá de la Excelencia y de la Calidad Total. México: Trillas.

- THOMPSON, Ivan. (2006). Definición de empresa. Disponible: http://www.promonegocios.net/mercadotecnia/empresa-definicionconcepto.html. Consultado: 15-10-11

- LEÓN, O.D y Honores, C.G (2003). Capital Social Juvenil y Evaluación Programática hacia jóvenes. Recuperado < http://dx.doi.org/10.4067/S0718-22362003000100009>. Consultado [ 28$1-14]$

- LOAYZA, Norman. (2010). Causas y consecuencias de la informalidad en el Perú. BCRP. Estudios económicos. Disponible: http://www.bcrp.gob.pe/docs/Publicaciones/Revista-Estudios-

Economicos/15/Estudios-Economicos-15-3.pdf. Consultado: 15-10-11.

- PÉREZ LEGOAS, Luis. (2002). Estadística básica para cienciassociales y educación. Lima. Editorial San Marcos.

- RAMOS, Ana Luz. (2006). ¿Qué es la economía Informal?. Disponible en: http://www.eumed.net/cursecon/ecolat/mx/2006/rsgb-informal.htm; Consultado: 16-10-11.

- VIGIL Lazo, Yolanda. (2010). Formación, crecimiento y proyección de las Micro y pequeñas empresas (MYPES). Disponible: http://www.monografias.com/trabajos58/mypes/mypes2.shtml. Consultado: 15-10-11.

- VELAZCO, Jackeline. ( 2004). La Protección Social para Trabajadores Informales en el Perú. Departamento de Economía. Pontificia Universidad Católica del Perú. Lima. Disponible: http://cies.org.pe/files/active/1/Wiego_Desco.pdf. Consultado: 15-10-11

- VAlDIVIA Dieñas, Raúl. (2009). Elaborando la Tesis. Tacna. Perú. Editorial UPT.

- WELLER, J. (2006). Inserción Laboral de Jóvenes: Expectativas, demanda laboral y trayectoria. < http://www.redetis.org.ar/media/document/weller.pdf>. Consultado [27-114]

Recibido: 2/6/2015

Aceptado para publicación:

$7 / 7 / 2015$ 